\title{
A Design Concept and Kinematic Model for a Soft Aquatic Robot with Complex Bio-mimicking Motion
}

\author{
Shokoofeh Abbaszadeh ${ }^{1}$ (]) $\cdot$ Roberto Leidhold ${ }^{1} \cdot$ Stefan Hoerner ${ }^{2}$
}

Received: 24 July 2021 / Revised: 3 November 2021 / Accepted: 14 November 2021 / Published online: 4 January 2022

(c) The Author(s) 2021

\begin{abstract}
Fish mortality assessments for turbine passages are currently performed by live-animal testing with up to a hundred thousand fish per year in Germany. A propelled sensor device could act as a fish surrogate. In this context, the study presented here investigates the state of the art via a thorough literature review on propulsion systems for aquatic robots. An evaluation of propulsion performance, weight, size and complexity of the motion achievable allows for the selection of an optimal concept for such a fish mimicking device carrying the sensors. In the second step, the design of a bioinspired soft robotic fish driven by an unconventional drive system is described. It is based on piezoceramic actuators, which allow for motion with five degrees of freedom (DOF) and the creation of complex bio-mimicking body motions. A kinematic model for the motion's characteristics is developed, to achieve accurate position feedback with the use of strain gauges. Optical measurements validate the complex deformation of the body and deliver the basis for the calibration of the kinematic model. Finally, it can be shown, that the calibrated model presented allows the tracking of the deformation of the entire body with an accuracy of $0.1 \mathrm{~mm}$.
\end{abstract}

Keywords Unconventional drive system $\cdot$ Biologically inspired robots $\cdot$ Biomimetics $\cdot$ Flexible robots

\section{Introduction}

According to the European Water Framework Directive, a risk assessment for fish injury and mortality in turbine passages is mandatory for innovative technologies, new installations, and existing hydropower facilities. State-ofthe-art methodologies to determine the risk of injury to fish in downstream turbine passages deploy live-animal tests, injecting wild fish of different species in the turbine. These live tests not only lead to serious injuries or even the death of the probands in many cases, as shown by Pracheil et al. [1], but are also very time and cost intensive. Alternative

Copyright@ S. Abbaszadeh, R. Leidhold, S. Hoerner 2021.

Shokoofeh Abbaszadeh

abbaszadeh@ovgu.de

1 Institute of Electrical Power Systems, Department of Electrical Engineering and Information Technology, OttoVon-Guericke University of Magdeburg, Universitätsplatz 2, 39106 Magdeburg, Germany

2 Institute of Fluid Dynamics and Thermodynamics, Department of Process and Systems Engineering, Otto-Von-Guericke University of Magdeburg, Universitätsplatz 2, 39106 Magdeburg, Germany methods are being investigated to replace or reduce the number of live fish tests, like the use of numerical studies, mainly based on computational fluid dynamics, such as those by Müller et al. [2], Klopries [3] or Gomez et al. [4], as well as deploying passive probes featuring pressure and acceleration sensors to determine the pressure drop and strike force events as a surrogate for fish, such as those carried out by Pauwels et al. [5], Tuhtan et al. [6] or Fu et al. [7]. However, a fish will neither behave as a dot, as it is commonly modeled in the numerics, nor as a passive body, drifting through a turbine like a wooden stick. As a consequence, up to now, these methods cannot yet provide sufficiently satisfying data to convince authorities and stakeholders that they are a viable replacement for live tests. Finding and initiating alternative methods as new standards for evaluating the risk of injury on a turbine downstream passage is the motivation for the development of a propelled soft robotic device. Here we present a snapshot of the ongoing development process towards such a robot.

The development of a suitable robotic surrogate system for deployment in such a harsh environment, equipped with sensors to sense the pressure and acceleration acting on the robot body, is challenging. Therefore, the focus of the study at hand is on the selection of a powerful, small 
and lightweight propulsion system for this surrogate, which shall allow for an efficient bio-mimicking body motion. The robot will have to meet the required biological similarity to the real probands and survive the harsh conditions during a turbine passage. This leads to very specific and particular requirements, which are subsequently defined in Sect. 2 .

The article features three major topics. The first contribution is a review of the abilities of commonly used propulsion systems and robotic fish designs with an emphasis on our particular requirements: the weight, swimming velocity, $\mathrm{BL} \mathrm{s}^{-1}$, DOF and weight to length ratio of the robotics. Then after evaluating the advantages and drawbacks of the propulsion system an unconventional actuator is chosen in accordance to the findings in Sect. 3. The second core topic is the presentation of the hardware implementation of the propulsion system best fulfilling our requirements (Sect. 4).

A general problem with studying the fluid-body interaction of such a soft robot with the fluid flow is the complex, non-linear feedback system of body motion and flow. It is not possible to gain knowledge about the real body motion without the use of an additional position feedback on the flexible part. For this reason, as the third key topic, we have developed a kinematic model which correlates the motion from point wise strain gauge measurements tracking two locations on the actuated body in Sect. 5. At first, the kinematic model for the complex, bio-mimicking motion with five DOF is derived. The position feedback system of the robotic propulsion system is calibrated. We present an approach based on optical measurements and classical image segmentation algorithms synchronized with the deployment of strain gauges.

Finally, in Sect. 6, the entire method is validated by the employment of arbitrary motion laws and the comparison of the model-based deformation prediction from the feedback system with the results of the optical measurements. Our contribution to the research community is the presentation and development of an unconventional actuator design for a bio-mimicking, multi DOF, lightweight and powerful propulsion system on a soft robotic device with accurate position feedback.

\section{Considerations and Requirements for a Robotic Fish Surrogate}

Fish feature different forms of locomotion, swimming power and speed depending on the species and the individual size. Generally, there exist two main categories of swimming modes: body and/or caudal fin locomotion (BCF) and median or pectoral fin locomotion (MPF). The BCF can be classified as anguilliform, carangiform, thunniform, ostraciiform or subcarangiform types [8, 9]. Salmonides, in this specific case brown trout, model species for the robotic device belong to the category of subcarangiform swimmers.

Limited size and weight as well as similar locomotion patterns are the primary challenge for all aquatic robots featuring neutral buoyancy. In consultation with the project's biologist, a brown trout was selected as a template for the robot. This species is a common model in ecohydraulic assessment studies. It is due to the typical body shape for rheophilic species and because these wild fish are quite easy to obtain, as they are relatively common in streams and rivers. Their good swimming ability and endurance allows them to swim against the current as an avoidance reaction and therefore remaining for a longer time in danger zones such as the turbine rotors. According to the authors' hypothesis, this could increase the probability of being hit by turbine blades compared to poor swimmers. As a consequence, a perfect surrogate for a brown trout would have a maximum length of $300 \mathrm{~mm}$. Furthermore, the robot may not be too heavy to remain neutrally buoyant. Which is an impossible challenge, considering the swimming speed to Body Length relation $\left(\mathrm{BL} \mathrm{s}^{-1}\right)$ of 6.6 with respect to the state-of-the art in aquatic robotics subsequently presented.

Archimedes' principle requires the robot to have the same density as water for neutral buoyancy. The maximum mass of the robot $m_{r}$ can thus be calculated according to:

$m_{r}=\rho_{w} V_{w}$,

where $V_{w}$ is the volume of the displaced water and $\rho_{w}$ is its density. According to Ebel [10], carangiform swimmers feature a relation between width $w$ and height $\mathrm{h}$ to the body length 1 of about 0.1 . In this case, the allowed mass can be roughly estimated to be $270 \mathrm{~g}$ for a $300 \mathrm{~mm}$ long robot:

$m=l w h \rho=0.01 l^{3} \rho$.

In 2013, Cen and Erturk [11] provided an overview of the body length to swimming speed relation for different actuator systems deployed in robotic fish application studies. According to this interesting report, conventional electrical machines, such as servos and brushless drives, are used to propel aquatic robots at high swimming speeds. However, none of the devices were small enough to be suitable as a brown trout surrogate. Furthermore, sparse information about the device's volume and buoyancy characteristics were provided, which are key for the application at hand. Subsequently, with respect to the available data, an actuator performance comparison will be investigated with a focus on the density ratio and DOF achievable in the swimming motion. 


\section{A Short Review of Aquatic Robotic Propulsion Systems}

Multiple thorough literature reviews on existing propulsion designs have been performed in the past, such as Cen \& Erturk [11], which provided an interesting overview of relative speed $\left(\mathrm{BL} \mathrm{s}^{-1}\right)$ related to body length. However, these reviews lack an explicit investigation of the key interests of this study, like the relation of relative speed (mostly expressed in $\mathrm{BL} \mathrm{s}^{-1}$ ) to weight and weight to body length. One major challenge for aquatic robots is neutral buoyancy, combined with acceptable size and swimming performance, which is often neither reached nor explicitly mentioned. In this case, additional floating devices or supports are necessary to compensate for negative buoyancy. However, besides a fish mimicking motion (expressed by high DOF), neutral buoyancy is crucial for the application at hand and those two are therefore key to the review. It must be noted that the robotic device cannot exceed $300 \mathrm{~mm}$ in body length as mentioned above. For this reason, both the relative and absolute values of length and weight are considered.

Figure 1 shows this comparison of conventional and unconventional propelled robotics with a focus on swim performance to density and available DOF for the motion pattern. Typical constructions use DC servomotors combined with transmission or gear systems, like levers, rods or cables, consisting of at least one, but often multiple segments [12-17]. Liu \& Hammond [17] used a cable-driven actuation mechanism designed as a cascaded skeletal structure with nine joints offering one DOF. This soft robot of $380 \mathrm{~mm}$ in length reached a maximum linear velocity of $0.135 \mathrm{~m} \mathrm{~s}^{-1}$ (approximately $0.35 \mathrm{BL} \mathrm{s}^{-1}$ ). There was no information provided about the weight of the entire device. However, the servo drive and skeleton mass was about $0.335 \mathrm{~kg}$. Behbahani \& Tan [12] built a device with a $150 \mathrm{~mm}$ body length and a weight of $0.3 \mathrm{~kg}$. They deployed three servo drives ( $3 \mathrm{DOF}$ ) propelling tail and caudal fins individually and reached a velocity of 0.3 $\mathrm{BL} \mathrm{s}{ }^{-1}$ at $2 \mathrm{~Hz}$ tail beat frequency. The dimensions of the robot used by Kodati et al. [18] led to a similar result of $0.27 \mathrm{BL} \mathrm{s}^{-1}$ with a weight of $0.49 \mathrm{~kg}$.

In 2014, $\mathrm{Wu}$ et al. [13] designed a $495 \mathrm{~mm} \times 50 \mathrm{~mm} \times 80 \mathrm{~mm}$ self-propelled subcarangiform robotic fish with four joints, operated by four DC servomotors to reach high maneuverability (4 DOF). Its mass was $1.29 \mathrm{~kg}$, and the device reached a remarkable maximum speed of $0.57 \mathrm{~m} \mathrm{~s}^{-1}\left(1.15 \mathrm{BL} \mathrm{s}^{-1}\right)$. Later, in 2019 , the group designed a $1.58 \mathrm{~m}$ long and of about $58.1 \mathrm{~kg}$ heavy gliding robotic dolphin. It could reach $0.405 \mathrm{~m} \mathrm{~s}^{-1}\left(0.25 \mathrm{BL} \mathrm{s}^{-1}\right)$ at a tail beat frequency of $2.5 \mathrm{~Hz}$ using two powerful brushless DC drives (2 DOF) [19]. The bio-mimetic carangiform swimming robotic fish created by Ay et al. [14] features two joints (5 DOF) driven by servomotors and obtained a speed of $0.4258 \mathrm{~m} \mathrm{~s}^{-1}\left(0.8516 \mathrm{BL} \mathrm{s}^{-1}\right)$ at $2 \mathrm{~Hz}$. This robotic fish is $500 \mathrm{~mm} \times 76 \mathrm{~mm} \times 215 \mathrm{~mm}$ in size and weighs about $3.1 \mathrm{~kg}$. The yellow-box-robotic fish of Wang et al. [15] was $400 \mathrm{~mm} \times 140 \mathrm{~mm} \times 142 \mathrm{~mm}$ in size and propelled by three

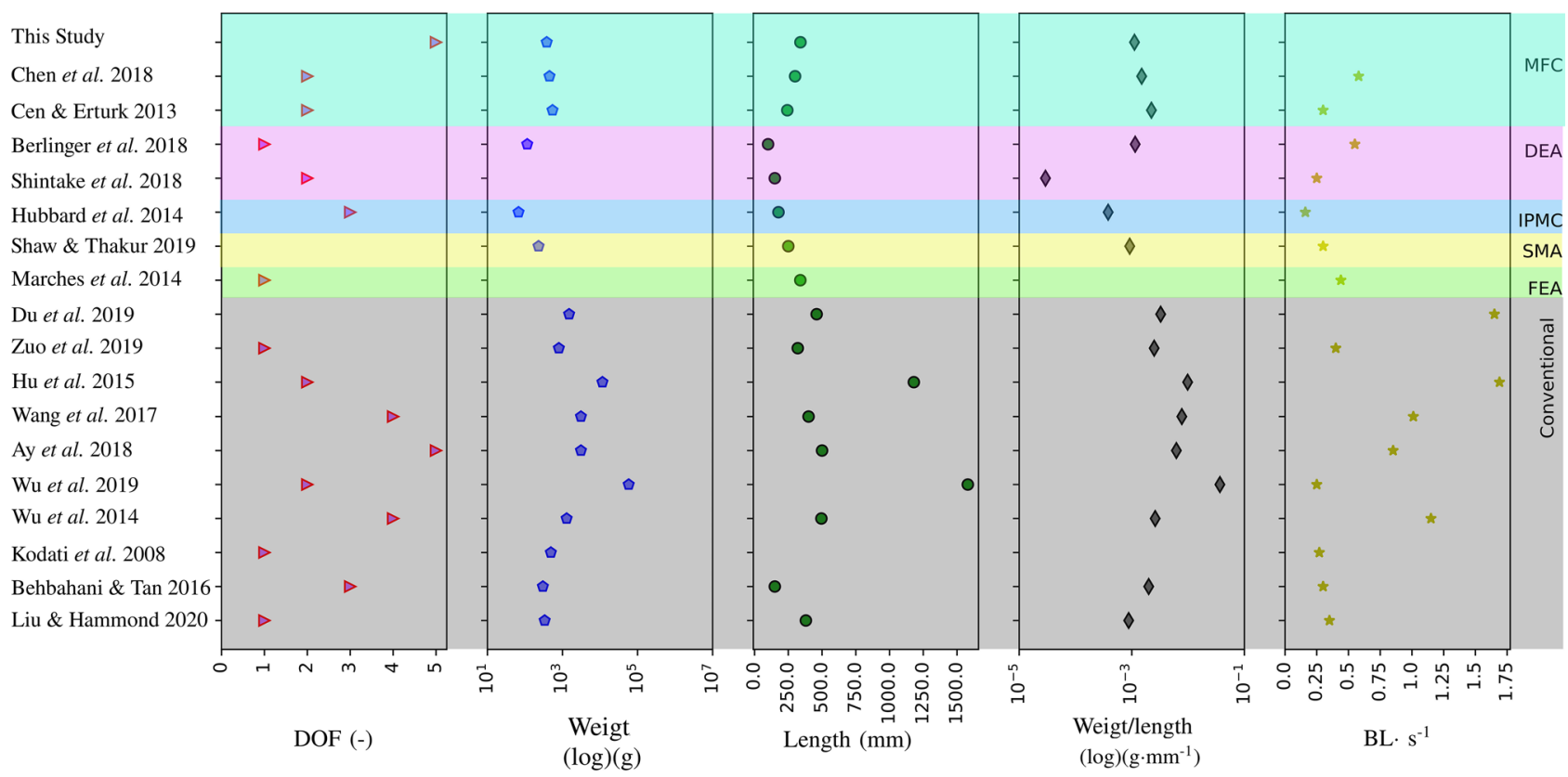

Fig. 1 Full characterization of the performance and technical specifications for conventional and unconventional propulsion systems according to the specific data available from seventeen recent pub- lications and the study presented here. From left to right, the DOF achieved, weight, length, weight-to-body length ratio and $\mathrm{BL} \mathrm{s}{ }^{-1}$ are compared 
servomotors. This $3.1 \mathrm{~kg}$ heavy robot was built to autonomously optimize a four DOF gait function for maximum swim velocity. It attained a maximum speed of $0.40 \mathrm{~m} \mathrm{~s}^{-1}$ (1.011 $\mathrm{BL} \mathrm{s}^{-1}$ ) at $2 \mathrm{~Hz}$ tail beat frequency. A subsequent study, introducing a novel methodology to track the robotic motion trajectory using an artificial lateral line systems showed that this fish robot had high maneuverability [20]. Hu et al. [21] presented a $1180 \mathrm{~mm} \times 330 \mathrm{~mm} \times 400 \mathrm{~mm}$ dolphin robot actuated by two DC brushless and two servo drives to individually control its fins. According to $\mathrm{Hu}$ et al., the device provided a 2 DOF motion. It achieved a maximum speed of $2.0 \mathrm{~m} \mathrm{~s}^{-1}$ (approximately $1.69 \mathrm{BL} \mathrm{s}^{-1}$ ) at a frequency of about $2.8 \mathrm{~Hz}$ while emulating a thunniform locomotion pattern. This is the fastest robotic swimmer to the authors' knowledge. The displacement of the robot is given as $11.6 \mathrm{~kg}$.

Zuo et al. [22] combined a servo driven tail (1 DOF) with a compact water electrolyser as a buoyancy control device to achieve a 3D maneuverable fish robot. The $320 \mathrm{~mm}$ long robot had a weight of $0.8 \mathrm{~kg}$ and reached $0.13 \mathrm{~m} \mathrm{~s}^{-1}$ $\left(0.4 \mathrm{BL} \mathrm{s}^{-1}\right)$ forward velocity. Du et al. [23] reached the remarkable relative speed of $1.65 \mathrm{BL} \mathrm{s}^{-1}$ (with a size of $460 \mathrm{~mm} \times 100 \mathrm{~mm} \times 130 \mathrm{~mm}$ ). The robot weighed about $1.5 \mathrm{~kg}$ and was actuated by two DC drives.

In conclusion, it can be stated that conventional actuators may allow for complex motion and the highest swim velocities but are not an acceptable mass for a small neutrally buoyant robot. Therefore, conventional methods were considered not to be suitable for the requirements of the project as stated above. Subsequently, an analysis of unconventional actuators for aquatic robots was performed. Soft actuators offer an unconventional solution for drive systems and are deployed in particular for propulsion systems in bioinspired and biomimetic underwater robots. These soft actuators are light, flexible and can be easily activated.

Marchese et al. [24] used Fluidic Elastomer Actuators (FEA) for a $339 \mathrm{~mm}$ long soft bodied robotic fish. According to the authors, FEA allow for an emulation of the slender anatomical form of a fish and to enable rapid escape responses. Elastomers are directly powered by pressurized fluid (1 DOF). This robot is able to swim forward at $1.67 \mathrm{~Hz}$ with linear velocity of $0.150 \mathrm{~m} \mathrm{~s}^{-1}\left(0.44 \mathrm{BL} \mathrm{s}^{-1}\right)$. These actuator types have a limited actuation bandwidth and are susceptible to failure by rupture. The weight of the device was not provided, but negative buoyancy was reported.

Shape memory alloy actuators (SMA) represent a type of smart materials which deform in response to temperature changes. SMA have the capability to memorize the shape defined during a setting operation and re-deform to this shape by heating it above a phase transformation temperature. Shaw \& Thakur [25] used a paired of $0.2 \mathrm{~mm}$ SMA wires embedded in a $0.6 \mathrm{~mm}$ flexible Polyethylene Terephthalate (PET) thermoplastic as the backbone and covered them with silicone. It propelled a robotic cuttlefish with overall dimension of $250 \mathrm{~mm}$ long, $37 \mathrm{~mm}$ in width and $60 \mathrm{~mm}$ in height. This $0.230 \mathrm{~kg}$ robot achieved a swimming speed of $0.076 \mathrm{~m} \mathrm{~s}^{-1}\left(0.3 \mathrm{BL} \mathrm{s}^{-1}\right)$. The SMA wires are heated with a pulse of electrical current with an adjusted phase shift and time offset to control the propulsion at the fin. According to Gupta et al. [26], SMA offers the advantage of low actuating voltage (under $12 \mathrm{~V}$ ) and a high power density. However, the slow response speed and low efficiency are two important disadvantages of this kind of actuator.

Electroactive polymers (EAP) such as ionic polymermetal composites (IPMC), dielectric elastomer actuators (DEA) and piezoelectric polymers are another group of unconventional actuators promising high force and powerto-density ratios. Therefore, this group of actuators is the focus of experimental research on soft robotics for underwater bioinspired and biomimetic robotics, even if they feature generally slower swimming speeds than SMA and FEA actuators. IPMCs are usually a sandwich composite of a thin electrolyte and two layers of noble metal as an electrode. Hubbard et al. [27] used an electrically driven IPMC built as an artificial muscle with uniquely patterned electrodes allowing for complex deformations and bioinspired locomotive behavior for their $177 \mathrm{~mm}$ long and $0.067 \mathrm{~kg}$ robot. It reached a multitude of DOF in an interesting and extensive experimental set up. Despite, this impressive effort, its average swimming speed of $0.028 \mathrm{~m} \mathrm{~s}^{-1}\left(0.16 \mathrm{BL} \mathrm{s}^{-1}\right)$ at $2 \mathrm{~Hz}$ and blocking force of $16.5 \mathrm{mN}$ at $3 \mathrm{~V}$ is still not enough to swim in turbulent flows. IPMC offers the advantage of a low driving voltage, high actuation frequency and a light and deformable construction, which leads to high compatibility for underwater use. However, a low actuating force and thus slow swimming speed are the drawbacks of this technology.

DEAs are commonly made from a carbon-based, metallic hydrogel or electrolyte electrodes and are flexible and feature high power density, high electromechanical transduction efficiency and have a density close to water [27]. Shintake et al. [28] used DEA for a biomimetic underwater robot. This robotic fish was $500 \mathrm{~mm} \times 400 \mathrm{~mm} \times 120 \mathrm{~mm}$ with a swimming speed of $0.0372 \mathrm{~m} \mathrm{~s}^{-1}\left(0.25 \mathrm{BL} \mathrm{s}^{-1}\right)$ at $0.75 \mathrm{~Hz}$ actuated at $5 \mathrm{kV}$. Berlinger et al. [29] developed a $100 \mathrm{~mm} \times 60 \mathrm{~mm} \times 30 \mathrm{~mm}$ 3D-printed robot with DEA actuators and a mass of $0.115 \mathrm{~kg}$. He measured the thrust force and swimming speed from 1 to $5 \mathrm{~Hz}$ up to approximately 25 $\mathrm{mN}$ and $0.055 \mathrm{~m} \mathrm{~s}^{-1}\left(0.55 \mathrm{BL} \mathrm{s}^{-1}\right)$. The actuators active area was limited to $20 \mathrm{~mm} \times 25 \mathrm{~mm}$ with applied voltage of $2 \mathrm{kV}$.

The challenges and limitations of these kinds of actuators are their high voltage actuation, which force the use of amplifiers along with a complicated and time-consuming process of fabrication to reach satisfying endurance and reliability. Dielectric charges with a voltage beyond the range 
of safe operation will cause short circuits in the material and lead to material degradation.

The last unconventional actuators evaluated in this study are macro-fiber composites (MFC), which are based on piezoelectric fiber composites. They feature high power density, large displacement, high level of strain and fast response. However, the non-linearity of the dynamic response has to be considered as an unwanted disturbance with respected to drive control accuracy and requires a closed loop control for precise position control. MFC consists of normally aligned rectangular piezoceramic fiber sheets and a structural epoxy sandwich between integrated electrode patterns on a polyamide film [30]. Like DEA, MFC need a high voltage input. They can be driven by a $2 \mathrm{kV}$ peak to peak driving input voltage (-500 VDC to $1500 \mathrm{VDC}$ ) without depolarizing of the dielectrics.

Cen \& Erturk [11] presented a robotic fish with two complementary actuated MFCs, a cantilevered MFC bimorph composed of two bonded single-layer MFC laminates, and a $1 \mathrm{kV}$ peak to peak driving input voltage. This robot could swim untethered at $0.075 \mathrm{~m} \mathrm{~s}^{-1}\left(0.31 \mathrm{BL} \mathrm{s}^{-1}\right)$ at $5 \mathrm{~Hz}$ tail frequency for a $242.6 \mathrm{~mm}$ body length with total mass of $0.541 \mathrm{~kg}$. Chen et al. [31] presented another MFC bimorphbased device actuated with $2 \mathrm{kV}$, which led to a swimming speed of $0.174 \mathrm{~m} \mathrm{~s}^{-1}\left(0.58 \mathrm{BL} \mathrm{s}^{-1}\right)$ in quiescent water for the $300 \mathrm{~mm}$ long robot. This untethered prototype had the form of a fish and a weight of $0.450 \mathrm{~kg}$. The displacement of the fixed MFC on the carbon fiber composite (CFC) plate increased monotonically along with actuating voltage and in a non-linear relation to the frequency. The characteristics of the MFC allow for variations in tail amplitude as a function of the peak voltage.

As a consequence of the findings reported in these various studies, it was considered that a MFC and DEA based propulsion system would best fit the requirements stated above and the MFC was chosen to perform some experiments.

Modeling a fish body's motion requires complex functions. According to Lou et al. [32], an exact kinematic of fish body movement is not achievable for a robotic device. Limitations are due to differences in parameters such as a different center of gravity, which leads to changes in head shaking. Starting with the slender-body-theory of Lighthill [33], a new model was proposed that offered complex motion with few DOF after simulation based improvements and subsequent experimental validation.

Figure 1 summarizes the literature review in this section. It can be concluded that conventional drive systems reach the highest $\mathrm{BL} \mathrm{s}^{-1}$ and generally higher velocities and higher DOF. However, their drawbacks are a poor weight-to-length ratio and negative buoyancy. Most unconventional propulsion systems lead to weak swimmers, but they offer more flexible and lighter bodies. Among unconventional drive systems besides DEAs, MFCs feature the highest swimming speeds with respect to $\mathrm{BL} \mathrm{s}^{-1}$. Considering the weight-tobody length ratio, DEAs and MFCs show the highest swimming speeds and offer more DOFs, even in comparison to conventional drive systems. In the study at hand, MFCs were deployed for actuation due to their availability at industrial quality and suitable size. However, DEAs may be a good alternative choice for this task.

Until now, soft underwater robot designs mostly offer only one or two DOF in their motion, regardless of type and amount of the actuators deployed. This paper presents a propulsion system design for soft robotics offering five DOF with the deployment of a combination of independently controllable unconventional MFC actuators. This allows for the creation of complex body movements for a neutrally buoyant soft underwater robot with limited size and fish alike shape.

\section{Hardware and Control}

\subsection{Mechatronical Design}

The robot's design consists of a rigid 3D-printed head, followed by a soft, actuated part and a passive tail fin as (a)

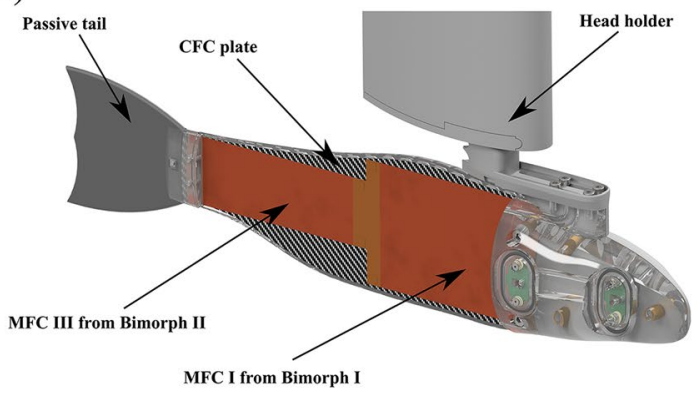

(b)

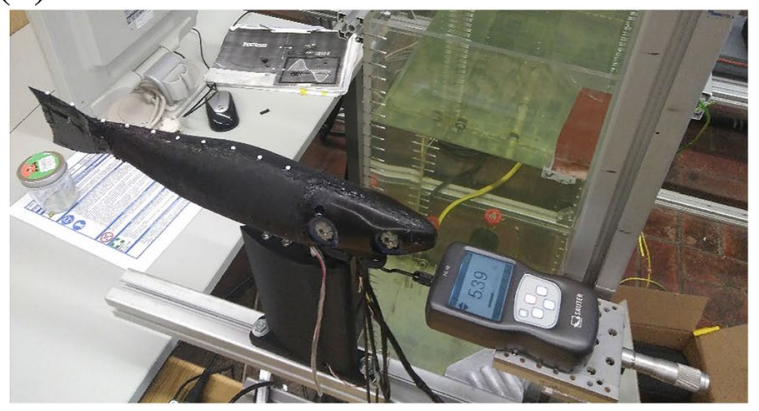

Fig. 2 (a) Design concept for the fish robot, including the 3D-printed head and mounting structure, and MFC actuators (in orange) on a CFC plate skeleton. b The fully assembled robotic device with flexible side embodiments from molded silicone 
shown in Fig. 2a. In the principal design, the skeleton and actuation are covered with two silicone embodiments with embedded air pockets providing a brown trout like shape (see Fig. 2b). For the optical measurements and the development of the kinematic models, the flexible embodiments were removed and neglected. The 100-mm long head section is equipped with cable channels and can be installed in a fixed or ball bearing pivot mount. The latter permits a head pitch, which is key for a bio-mimicking propulsion. The flexible, moving part, subsequently referred to as the body, is made of a $0.2 \mathrm{~mm}$ thick carbon fiber composite (CFC) plate, acting as a $182 \mathrm{~mm}$ long spine. Four piezoceramic actuators (MFC), in an arrangement of two couples of different sizes and performance, are placed on both sides of the CFC plate, forming a morphing structure. The $60 \mathrm{~mm}$ passive tail consists of a CFC plate covered with a silicone coating. Details of the actuators deployed can be found in Table 1 .

The entire body is waterproofed with a silicone finish, power supply, amplification and controller are placed externally at the current state for convenience and simplification of the experimental setup. In a later state of development, the robot is intended to become partly autonomous. Fortunately, the time scale for turbine passages is within seconds, and the minimal operation time for the device is therefore extremely short. Therefore, the power supply will only consist of $4 \mathrm{Li}$-ion cells with a total capacity of $2 \mathrm{Wh}$ and a total mass of $12 \mathrm{~g}$. This leads to a run time of approximately $24 \mathrm{~min}$. The much higher capacity was chosen to ensure an extended measurement time.

Table 1 Hardware components technical specifications

\begin{tabular}{llll}
\hline CFC plate & CERP & & \\
\hline Young's modulus & $E$ & $(\mathrm{~Pa})$ & $60 e^{9}$ \\
Density & $\rho$ & $\left(\mathrm{Kg} \mathrm{m}^{-1}\right)$ & 0.156 \\
Fiber orientation & & $\left({ }^{\circ}\right)$ & $0 / 90$ \\
MFC-actuator & & & \\
Actuator I \& II & M-8557P1 & & \\
Actuator III \& IV & M-8525P1 & & \\
Free strain & & $(\mathrm{ppm})$ & 1350 \\
Operational volt- & & $(\mathrm{V})$ & -500 to +1500 \\
$\quad$ & & \\
age & & & PWM voltage amplifier AMD2012-CE3 \\
Control input & & (VDC) & 8 to 15 \\
Supply voltage & & $(\mathrm{V})$ & 0 to 2000 V \\
Output voltage & & & with 500 V \\
& & & bias \\
MCU & & & \\
Strain gauge & MI-F28069 & & \\
\hline
\end{tabular}

\subsection{Propulsion Control Concept}

The concept is based on two arrays of two MFCs, each of which is coupled as a complimentary actuator (bimorphs) on the two sides of the CFC plate. This allows for the creation of two groups of artificial muscles. In our case, they are controlled individually with a sinusoidal control signal (Eqs. (3) and (4)). Any other control signal would also be possible. Our setup allows the generation of a propulsion motion with five DOF overall. These consist of two amplitudes $\left(A_{1} \& A_{2}\right)$ and two propulsion frequencies $\left(f_{1} \& f_{2}\right)$, which can be individually set for each bimorph actuator (muscle group). The fifth DOF is provided by a phase shift in between the two sinusoidal functions.

The four actuators can be driven individually with a set of four high voltage power amplifiers. In the current setup, AMD2012-CE3 amplifiers (Table 1) from the actuator's vendor are deployed. This type of amplifier provides a variable output from $-500 \mathrm{~V}$ to $+1500 \mathrm{~V}$. Control is provided by a PWM signal with 1 to $2 \mathrm{~ms}$ pulses and a $5 \mathrm{~ms}$ period in $3.3 \mathrm{~V}$ logic. This signal is supplied by a microcontroller of type F28069 from Texas Instruments on which the control algorithm is implemented. Due to the hardware configuration, it is important to drive the actuator couples-placed on opposite sides as a bimorph - in a complementary fashion to reach a reasonable charge without damage. Software implemented voltage limitations prevent the MFC from depolarizing the dielectrics. This leads to a set of two independent functions for the actuation variables:

$X_{1}=A_{1} \sin \left(2 \pi f_{1} t\right)$,

$X_{2}=A_{2} \sin \left(2 \pi f_{2} t+\omega\right)$.

Figure 3 shows an example of complementary driving signals for bimorphs. However, open loop control does not allow for precise knowledge of the real motion performed by the robot. Damping and bending resistance from the skeleton and the embodiment are unknown parameters. The water will act as an added mass; further, non-linear and complex fluid-body interactions with vortices and flow structures are expected. These will depend not only on the general shape of motion, but also the frequency, amplitude and swimming speed. It is therefore necessary to track the real deformation of the CFC plate, subsequently referred to as the spine and on its impact on the entire body motion.

\subsection{Strain Measurement Setup}

Unfortunately, MFCs can be used either as an actuator or as a sensor. To achieve the maximum achievable amplitude and force, it makes sense to use both MFCs as an actuator in each bimorph and not to actuate one side while its 

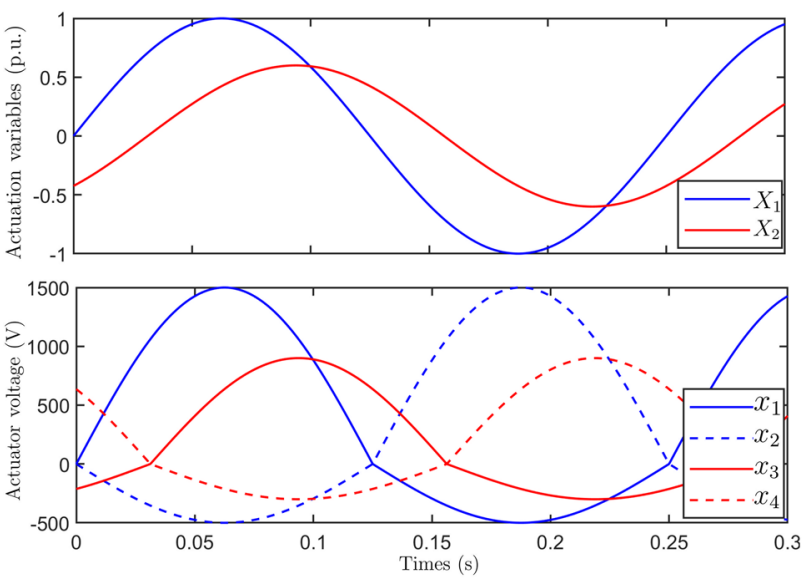

Fig. 3 (Top) Example plot for the control signal of the power amplifiers. (bottom) Signals $x_{1}$ and $x_{2}$ are complementary. This signal has been used for both actuators, while bimorph I is governed by $x_{1}$ and $x_{2} . x_{3}$ and $x_{4}$ provide the phase shifted signal for bimorph II with independent amplitudes and frequencies for bimorph I and II

counterpart senses the strain. The exact position feedback can be achieved by tracking the actuated strain on the skeleton. As will be shown later, this requires knowledge of only two points of the kinematic system, which are located on each of the bimorph actuators. As a consequence, the strain gauges were installed on each bimorph and arranged as full bridges. This results in two data sets for the entire length of the flexible part of the robotic fish (Fig. 4a). It allows for a correlation between the voltage signal from the strain gauge and the injected driving voltage. However, this will still not provide any information about the deflection of the flexible body. Therefore, a kinematic model must be developed and calibrated.

\section{Kinematic Model}

To drive the robot's kinematic model, it is assumed that the body is fixed on the head side. Thus, it behaves as a clamped beam. The remaining part can be considered to be built from three independent segments as shown in Fig. 4b. Four actuators, combined as two couples, of which each couple comprises a bimorph, are deployed: while the actuator on one side expands, the complementary actuator will contract. According to the Euler-Bernoulli beam theory, the CFC plate in the middle acts as the beam's neutral fiber, which is further assured by the CFC's high Young's modulus. Therefore, the middle segment of the CFC plate is considered to be constant. The elongation of the actuator on one side, combined with the contraction of its counterpart on the other, will lead to a circular bending of the body in the area of the two bimorph sections while they are actuated. The circular bending depends on the strain on the surface, or in this case the actuators, resulting in radii $r_{1}$ and $r_{2}$ respectively. They depend on the spine width $b$ as well as the strain $\epsilon_{1}$ and $\epsilon_{2}$ of both active sections. The two active parts are each of a length $\mathrm{L}=85 \mathrm{~mm}$. In between the two actuated areas, a passive part remains. This stiff part acts as the third segment, has a length of $\mathrm{z}=18 \mathrm{~mm}$ and behaves linearly.

The model is based on three frames of reference $\{x, y\}$, $\left\{x^{\prime}, y^{\prime}\right\}$ and $\left\{x^{\prime \prime}, y^{\prime \prime}\right\}$, which correspond to each segment as shown in Fig. 4b. In summation, this leads to three interdependent coordinate systems of superposed motion defined by a two-dimensional rotation matrix derived from the previous segments.

As a consequence, the position of a spine point in the xyplane can be described by a parametric equation of the arc length $l$ as follows:

for $0 \leq l \leq \mathrm{L}$ (segment I, bimorph I):

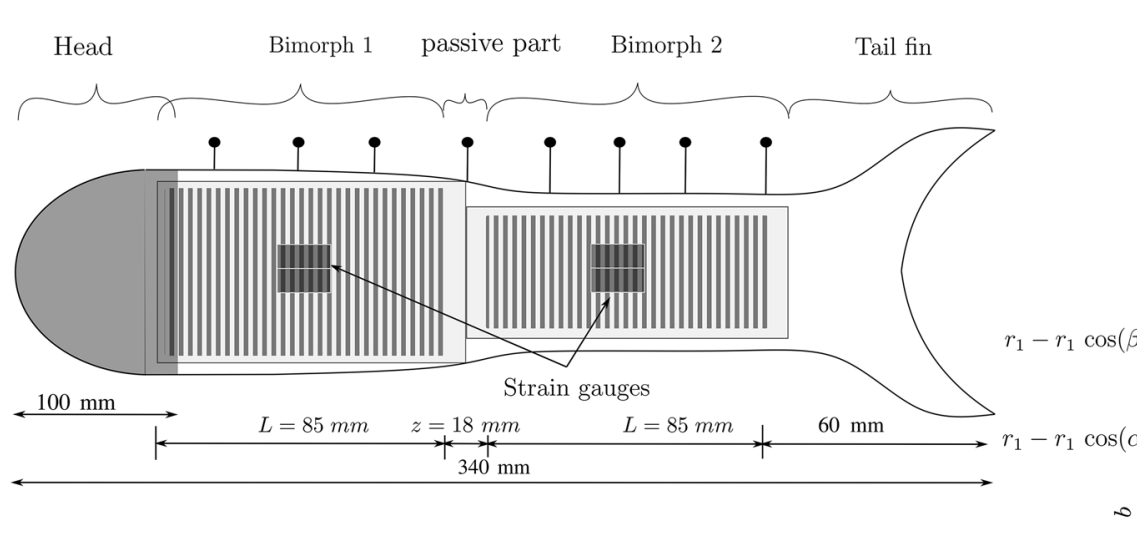

(a)

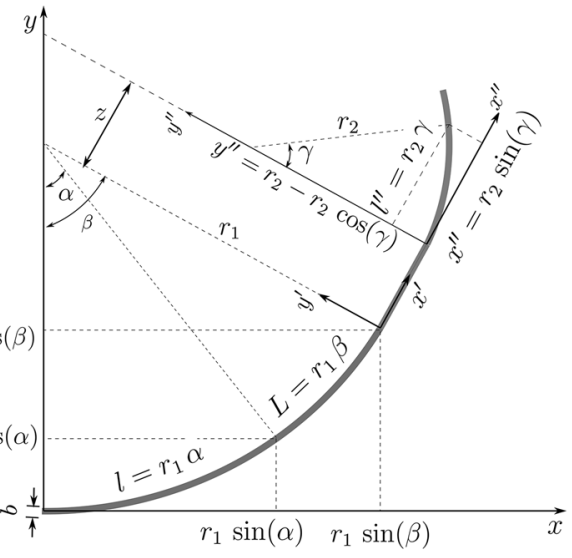

(b)

Fig. 4 (a) Concept for the robotic fish including actuators and strain gauges. (b) The body bending kinematic model 
$\left[\begin{array}{l}x \\ y\end{array}\right]=\left[\begin{array}{c}r_{1} \sin \alpha \\ r_{1}-r_{1} \cos \alpha\end{array}\right]$

with $\alpha=\frac{l}{r_{1}}$ and $r_{1}=\frac{b}{\epsilon_{1}}$

for $\mathrm{L} \leq l \leq \mathrm{L}+\mathrm{z}$ (segment II, passive):

$\left[\begin{array}{l}x \\ y\end{array}\right]=\left[\begin{array}{cc}\cos \beta & -\sin \beta \\ \sin \beta & \cos \beta\end{array}\right]\left[\begin{array}{c}l-\mathrm{L} \\ 0\end{array}\right]+\left[\begin{array}{c}r_{1} \sin \beta \\ r_{1}-r_{1} \cos \beta\end{array}\right]$

with $\beta=\frac{\mathrm{L}}{r_{1}}$

for $l>\mathrm{L}+\mathrm{z}$ (segment III, bimorph II):

$\left[\begin{array}{l}x \\ y\end{array}\right]=\left[\begin{array}{cc}\cos \beta & -\sin \beta \\ \sin \beta & \cos \beta\end{array}\right]\left[\begin{array}{c}x^{\prime \prime}+z \\ y^{\prime \prime}\end{array}\right]+\left[\begin{array}{c}r_{1} \sin \beta \\ r_{1}-r_{1} \cos \beta\end{array}\right]$,

where $\left(\begin{array}{c}x^{\prime \prime} \\ y^{\prime \prime}\end{array}\right)=\left(\begin{array}{c}r_{2} \cdot \sin \gamma \\ r_{2}-r_{2} \cdot \cos \gamma\end{array}\right), \gamma=\frac{l-\mathrm{L}-z}{r_{2}}$ and $r_{2}=\frac{b}{\epsilon_{2}}$

Selecting Eqs. (5)-(7) depending on the location of point 1 , the $x-y$ position can be summarized as a function of the length and thickness of each section as well as the strains:

$\left[\begin{array}{l}x \\ y\end{array}\right]=\left[\begin{array}{l}f_{x}\left(l, \epsilon_{1}, \epsilon_{2}\right) \\ f_{y}\left(l, \epsilon_{1}, \epsilon_{2}\right)\end{array}\right]$.

The dependence of the strain with respect to the voltage outputs of the strain gauges $u_{1}$ and $u_{2}$ is given by:

$\epsilon_{i}=k_{i} \cdot\left(u_{i}-u_{\text {off } i}\right), i=1,2$

Inserting these in Eq. (8), it yields:

$\left[\begin{array}{l}x \\ y\end{array}\right]=\left[\begin{array}{l}f_{x}\left(l, u_{1}, u_{2}, P\right) \\ f_{y}\left(l, u_{1}, u_{2}, P\right)\end{array}\right]$,

where $P=\left\{k_{1}, k_{2}, u_{\text {off } 1}, u_{\text {off } 2}\right\}$ is a parameter set of the strain gauge.

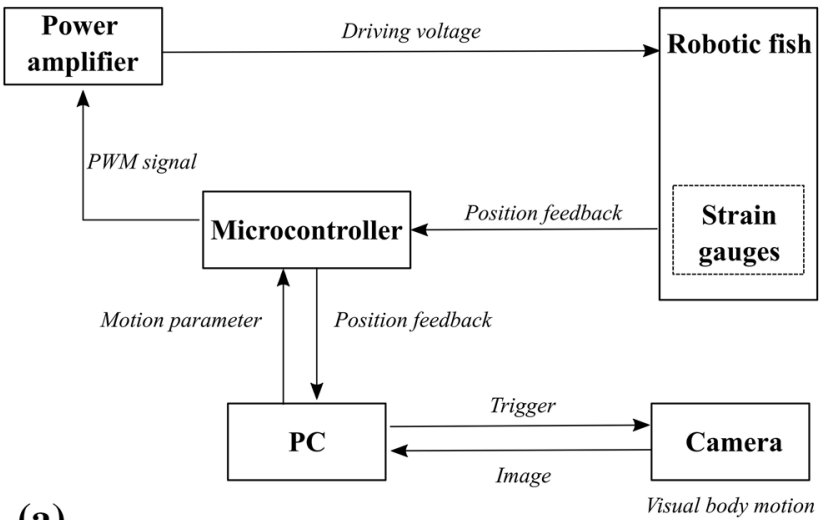

Fig. 5 (a) Signal flow diagram of the experimental setup for the optical measurements. (b) Experimental set up. (c-e) Three stages of the image processing are shown: (c) the original image from the camera,
To determine the strains $\epsilon_{1}$ and $\epsilon_{2}$ from the strain gauge signal, an experiment with optical measurements was deployed to gain information about the displacement $y(l)$ for each segment.

\section{Optical Measurements}

\subsection{Experimental Setup}

Figure 5 shows the signal flow chart and the schematics of the experimental setup. Eight white pin heads were fixed with equidistant spacing of $22.5 \mathrm{~mm}$ on the CFC plate (spine) as shown in Fig. 5 (middle). They allow for the recognition of the spine position. A Gopro Hero 9 camera $\left(5184 \times 3888 \mathrm{px}^{2}\right)$ installed on top of the body at a distance of $140 \mathrm{~mm}$ captured the spine's deformation. The aberrations from the camera lens were adjusted with the inbuilt lens calibration function provided by the manufacturer. The remaining error was considered to be negligible after the analysis of a sample image. The camera's acquisitions were synchronized with the amplifier signal via Python scripts. This allowed for the automated capture and processing of the images. All recordings were performed on quasi-static actuated deformations (static actuation $>12 \mathrm{~s}$ ). After the first evaluation, it was found that the skeleton would not return to its neutral position, due to hysteresis effects from the actuators. However, this only happens in static setups or in the case of nonsymmetrical movements. To eliminate these effects, a symmetrical periodic motion, here a damped sinusoidal oscillation function, had to be applied after each static measurement. Also, it must be noted that the actuators showed a slow creeping character in the very last part of the motion, while high amplitude set points were reached faster than was the case with lower amplitudes. For the

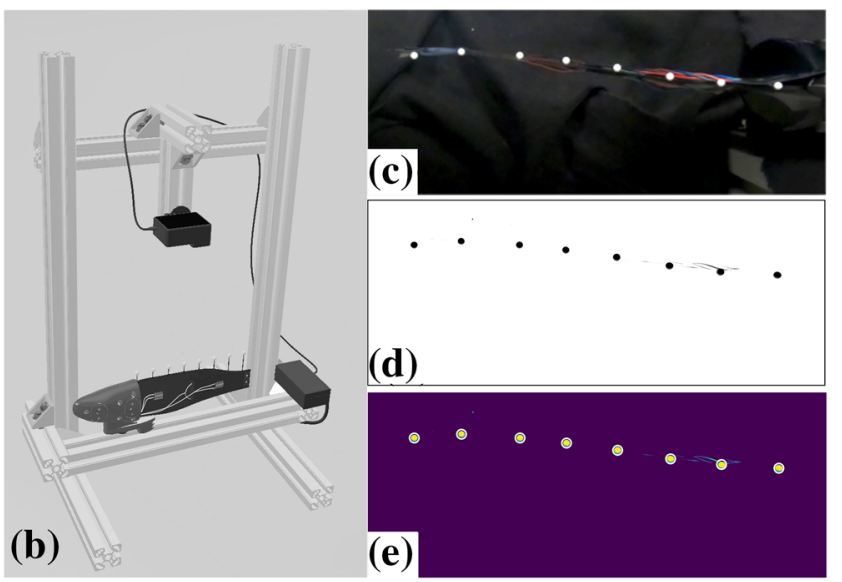

(d) the pretreated image for improved point recognition and (e) the correlation control image for visual control of the custom segmentation and correlation algorithm 
optical measurements, the analysis of the raw footage was performed with classical image segmentation algorithms taken from the scikit-image and SciPy libraries as well as correlation tools from the NumPy package. After a contrast enhancement, as the first step, a circular kernel the size of a pin head (40 px) was deployed for cross-correlation over a predefined detail of the image. For the second step, the correlation map was subsequently filtered by a threshold. The pin heads, the eight regions of the highest correlation peaks, were captured by a scipy.label function. Subsequently, the pin heads' center of the gravity was determined to deliver their coordinates.

\subsection{Kinematics Function Regression}

The information on the optical measurements in line with the synchronized strain gauge signal allow for a correlation of strain to motion and the derivation of a kinematic function with the use of the kinematic model.

As expressed in Eq. (9), $\epsilon_{1}$ and $\epsilon_{2}$ are linearly dependent relative to the output voltage of the strain gauges. Therefore, the following objective function (11) derived from Eq. (10) was minimized to determine $k_{1}$ and $k_{2}$ with help of the optimization algorithm toolbox in Matlab:

$\min _{P} \sum_{n=1}^{8} \sum_{m=1}^{M}\left(y_{\text {meas }, n, m}-f_{y}\left(l_{n}, u_{1 m}, u_{2 m}, P\right)\right)^{2}$,

where $\mathrm{y}_{\text {meas,n,m }}$ is the measured displacement, $\mathrm{n}$ is the marker number and $\mathrm{m}$ is the test number with a given actuator excitation of $\left\{X_{1 m}, X_{2 m}\right\}$.

Constraints were defined for the rotation angles of each segment to be less than $45^{\circ}$. This ensured a unique solution for each pin head position. The maximum voltage with respect to the offset is $u_{i}=u_{\max }=1.65 \mathrm{~V}$. Combining and solving for the gain it yields: $k_{\text {imax }}=\frac{b \beta}{u_{\text {imax }} \mathrm{L}}=0.0081$

The measurements used to obtain the data for the regression of the kinematics function were performed in three steps. At first, only bimorph I was actuated and observed in twenty static positions from 0 to $100 \%$ of the driving voltage $X_{1}$. In this setup, bimorph II was not actuated and behaved passively. The output voltages of the strain gauge's full bridges were recorded and stored as csv-data. As the second step, the same setup was repeated for bimorph II, while bimorph I acted as a passive part. In the last step of the experiment, both bimorphs were actuated. Several equal and opposite morphing positions were implemented.

Three different optimization models were deployed in Matlab, starting with fminsearch, which deploys the simplex search method of Lagarias et al. [34], up to genetic algorithms and a particle swarm optimization, without significant deviations.
In theory, the gain parameters $k_{1,2}$ have to be constant for all measurements. The following values were determined by a converged solution after 92 solver iterations over the kinematics function:

$\epsilon_{1}=0.0029\left(u_{1}-1.4464\right)$,

$\epsilon_{2}=0.0025\left(u_{2}-1.6156\right)$.

Knowledge of the kinematics function between the bending kinematics and strain gauges' voltage allows for the determination of the body's displacements without optical measurement.

\subsection{Results and Discussion}

During the quasi-static tests, the spin's tail pin head marker reached a displacement of about $33 \mathrm{~mm}$ in one direction. This can be interpolated to the additional length of the absent tail fin to about $50 \mathrm{~mm}$ and adds up to a total motion of about $100 \mathrm{~mm}$ in air. The optical measurement results and the corresponding voltage signal of the strain gauge's full bridges are displayed in Fig. 6. For each of the three measurement sets comprised at twenty actuator excitation variations, the measured voltage signal and calculated displacement is shown, at first for a single actuation of MFC I, then for a single actuation of MFC II, and subsequently for both MFC I \& II. Here it can be seen that the first point near the clamping position at the head hardly moves at all, while the last point at the end of active part reached a maximum displacement of almost $33 \mathrm{~mm}$ at $u_{1}=0.4 \mathrm{~V}$ and $u_{2}=1.73 \mathrm{~V}$ strain gauge signals.

Figure 6 allows for the evaluation of the kinematics function. In the subfigures, the red solid lines show the spine displacement calculated from the strain gauge signals with Eq. (10). The eight markers on each solid line present the displacement of the pin heads captured by optical measurements. Even if these points are not fully aligned, very good accordance can be noted.

Sources of errors can be found in the inaccuracies of the strain gauges and amplification, which affect the determination of the kinematics function and in optical measurement errors. The accuracy of the optical method was tested in a similar setup and found to have a RMS error of $0.007 \mathrm{~mm}$ or 0.11 pixels. The maximum deviation from the mean value was $0.12 \mathrm{~mm}$. For this purpose, ten sequential pictures of the setup without any actuation were captured and processed.

While the first two steps of the experiment provided the information to calculate $\epsilon_{1}$ and $\epsilon_{2}$, the third step of the experiment was performed to serve as a proof of concept. Figure 6 (bottom right) shows the arbitrary motions from the combination of bimorph I and II.

To evaluate the ability of the kinematics function derived earlier, a second set of test data comprising several bending 
Fig. 6 Displacement of the pin head markers as a function of the position along the spine, shown for three sets of quasistatic motions. The displacement (ordinate) is shown as a function of the length (abscissa) for unequal scaling of the axis to achieve better visualization. $A_{1}$ and $A_{2}$ are scaled from -1 to 1 , while -1 and 1 stand for the maximum opposing amplitudes in the actuator amplification (see Eqs. (3) and (4)); $\mathrm{u}_{1}$ and $\mathrm{u}_{2}$ is from 0 to $3.3 \mathrm{~V}$, while $1.65 \mathrm{~V}$ means no strain. (a) only bimorph I actuated (twenty-one variations), (b) only bimorph II actuated (twenty-one variations). (c) both bimorph I and II are actuated with arbitrary set points (twelve variations)
$Y$-position as function of $l$ (the marker position along the spine)

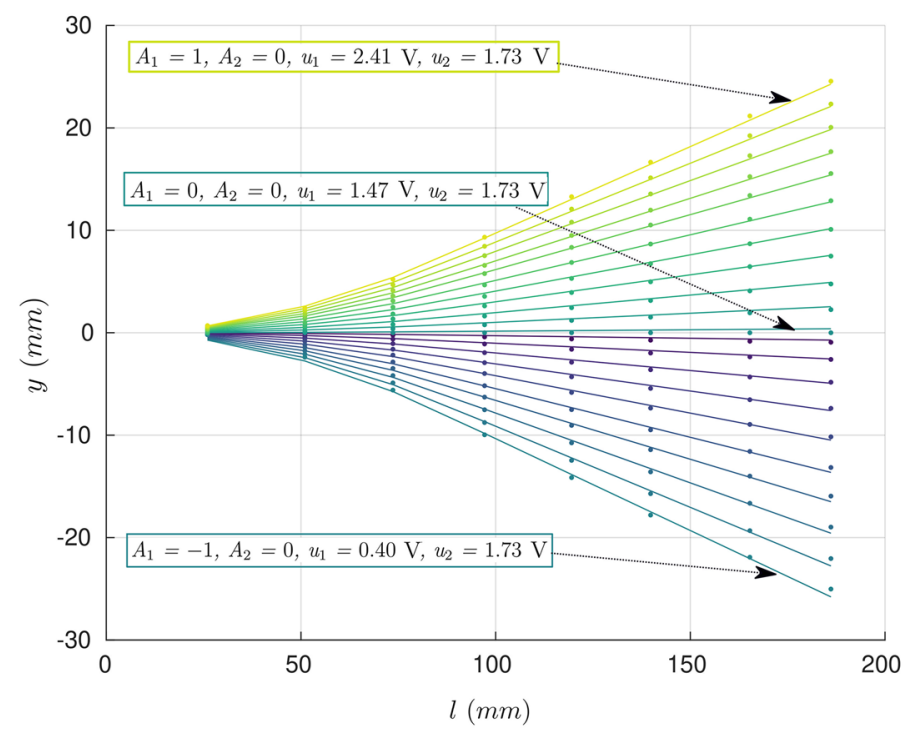

(b)

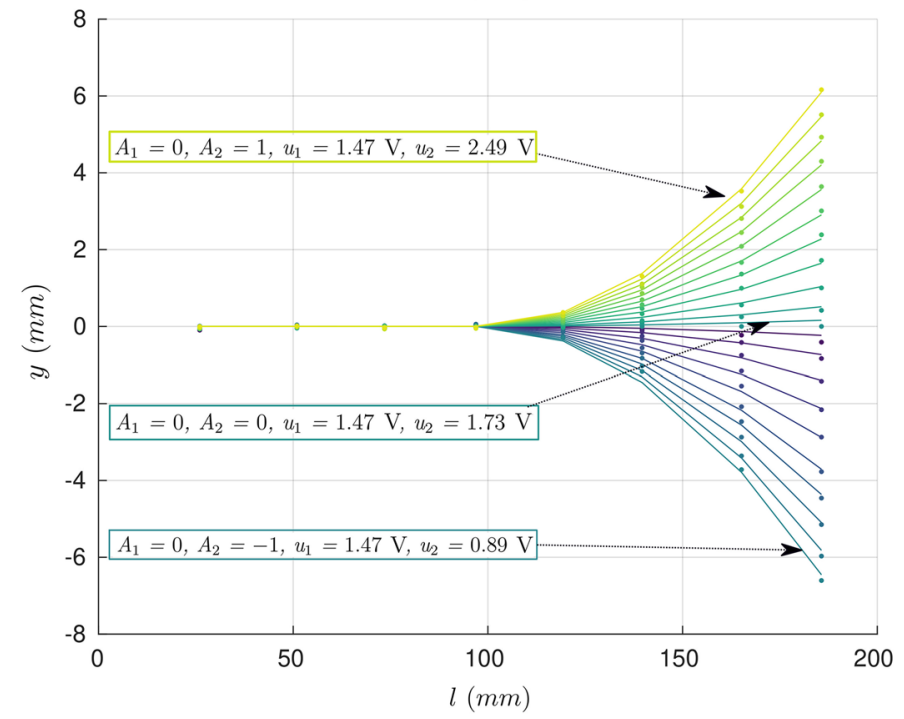

(c)

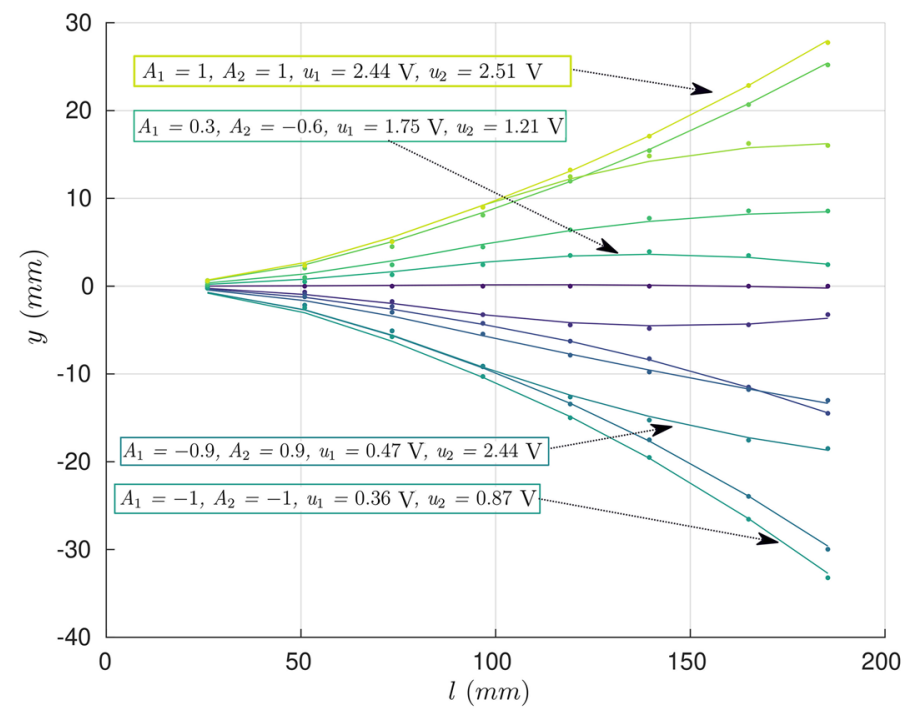




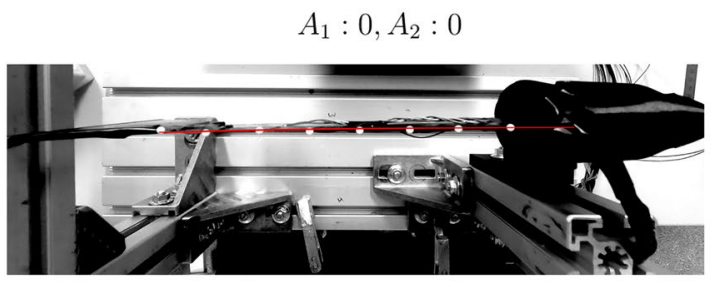

$A_{1}:-0.6, A_{2}: 0.3$

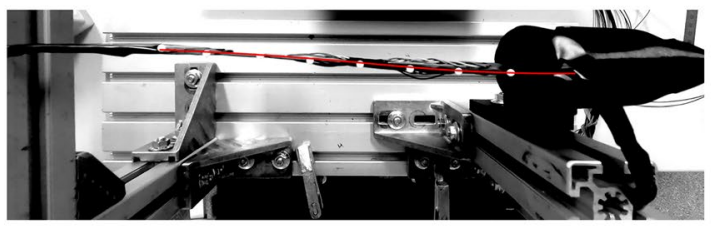

$A_{1}: 1, A_{2}: 1$

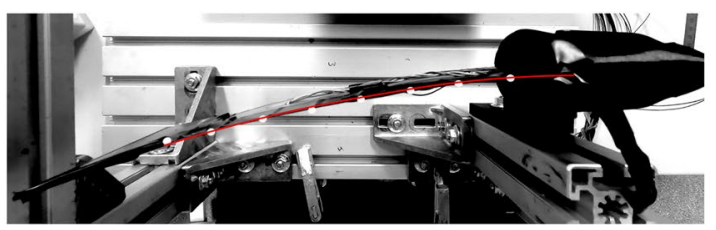

$A_{1}: 0.3, A_{2}:-0.6$

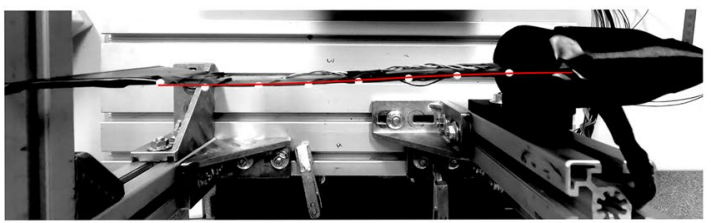

$A_{1}:-0.9, A_{2}: 0.9$

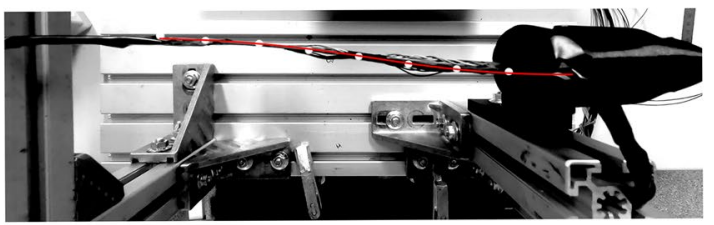

$A_{1}:-0.4, A_{2}: 0.9$

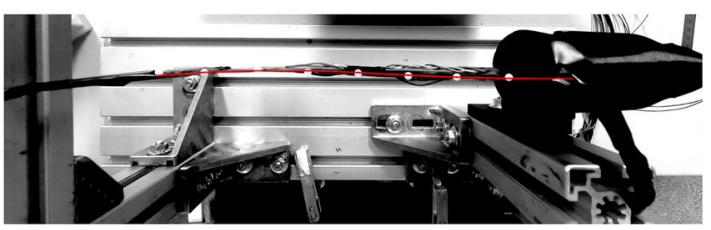

Fig. 7 Validation of the kinematics function for arbitrary motions. The motion is captured in the same setup as presented in Fig. 5. The red line is a plot of the curve derived from the kinematic model based on strain gauge measurements. $\left(A_{1}\right.$ and $A_{2}$ are according to Fig. 6)

situations was collected. For this purpose, once again quasistatic motions of both bimorphs were generated. The strain gauges' signal and a picture of the bent spine were saved on a PC. Splines created by use of the strain gauge signal and the kinematics function Eq. (10) were plotted in superposition on the corresponding image. This allows for a direct visual inspection of the modeled and captured displacements. Figure 7 shows the results of this process. It can be seen that the method provides precise position feedback with a high degree of accuracy.

\section{Conclusion}

A thorough literature review was performed for both conventional and unconventional propulsion systems used for bio-mimicking, aquatic robots. This evaluation clearly shows the need for an unconventional propulsion system, to best fit the particular requirements for the replacement of fish in live-animal tests. The selection, piezoelectric ceramic actuators, represents the best trade-off for the design of a neutrally buoyant, lightweight and small robotic device. They allow for a multiple DOF motion, an acceptable weight-to-length ratio and the highest relative swimming performance. The concept presented here features a soft robotic fish of $340 \mathrm{~mm}$ in length. It consists of a 3D-printed rigid head and a soft, actuated body equipped with multiple artificial muscles. The design allows for a motion with five degrees of freedom and the generation of an arbitrary fish mimicking motion. A motion amplitude of about $100 \mathrm{~mm}$ at the passive tail without any further deployment of gear or transmission systems is achieved. However, in this system, a challenge remains in the form of an accurate position feedback for the body's motion. To determine the complex deformation of the body, pointwise measurements are employed at two locations on the actuator couples. A kinematic model has been developed to predict the body's displacement. An experimental set up composed of optical measurements and custom processing routines based on image segmentation has been developed. This allows the determination and calibration of the kinematic model of the complex body motion, which has finally been validated. The model is able to feed back the entire body deformation with a high precision of about $0.1 \mathrm{~mm}$. This setup allows for detailed knowledge of the resulting motion and of the complex fluid-body interactions. Future research will be comprised of extended underwater tests and an optimization of the propelling body motion for the robot.

Acknowledgements The authors would like to thank Christian-Toralf Weber (University of Applied Sciences Magdeburg) for his advice, Andreas Bannack (OvGU) for his support in the implementation of MCU, Yanneck Kiiski (OvGU) for his assistance with the CAD modeling of the robotic prototype and Iring Kösters (OvGU) for his assistance developing the optical measurement setup. The remarks and comments from Jeff Andrew Tuhtan and Gert Toming (Centre for Biorobotics at Taltech, Estonia) are gratefully acknowledged. Furthermore, the authors would like to thank Falko Wagner (IGF Jena) for his support in answering biological questions. 
Funding Open Access funding enabled and organized by Projekt DEAL. This work was part of the RETERO project (https://retero.org). S.A. was funded by the German Ministry of Education and Research (BMBF) with grant number 031L0152A.

\section{Declarations}

Conflict of interest The authors declare no competing financial and non-financial interests.

Open Access This article is licensed under a Creative Commons Attribution 4.0 International License, which permits use, sharing, adaptation, distribution and reproduction in any medium or format, as long as you give appropriate credit to the original author(s) and the source, provide a link to the Creative Commons licence, and indicate if changes were made. The images or other third party material in this article are included in the article's Creative Commons licence, unless indicated otherwise in a credit line to the material. If material is not included in the article's Creative Commons licence and your intended use is not permitted by statutory regulation or exceeds the permitted use, you will need to obtain permission directly from the copyright holder. To view a copy of this licence, visit http://creativecommons.org/licenses/by/4.0/.

\section{References}

1. Pracheil, B. M., DeRolph, C. R., Schramm, M. P., \& Bevelhimer, M. S. (2016). A fish-eye view of riverine hydropower systems: the current understanding of the biological response to turbine passage. Reviews in Fish Biology and Fisheries, 26, 153-167.

2. Müller, S., Cleynen, O., Hoerner, S., Lichtenberg, N., \& Thévenin, D. (2018). Numerical analysis of the compromise between power output and fish-friendliness in a vortex power plant. Journal of Ecohydraulics, 3, 86-98.

3. Klopries, E. M., \& Schüttrumpf, H. (2020). Mortality assessment for adult European eels (Anguilla Anguilla) during turbine passage using CFD modelling. Renewable Energy, 147, 1481-1490.

4. Romero-Gomez, P., Lang, M., Michelcic, J., \& Weissenberger, S. (2019). Particle-based evaluations of fish-friendliness in Kaplan turbine operations. IOP Conference Series: Earth and Environmental Science, 240, 42016.

5. Pauwels, I. S., Baeyens, R., Toming, G., Schneider, M., Buysse, D., Coeck, J., \& Tuhtan, J. A. (2020). Multi-species assessment of injury, mortality, and physical conditions during downstream passage through a large archimedes hydrodynamic screw (Albert Canal, Belgium)". Sustainability, 12, 20.

6. Tuhtan, J.A., Fuentes-Perez, J.F., Angerer, T., Schletterer, M. (2018) Monitoring upstream fish passage through a bypass pipe and drop at the fish lift Runserau: Comparing dynamic pressure measurements on live fish with passive electronic fish surrogates. 12th ISE 2018 International Symposium of Ecohydraulics, Tokyo, Japan, 2018.

7. Fu, T., Deng, Z. D., Duncan, J. P., Zhou, D. Q., Carlson, T. J., Johnson, G. E., \& Hou, H. (2016). Assessing hydraulic conditions through Francis turbines using an autonomous sensor device. Renewable Energy, 99, 1244-1252.

8. Low, K. H., \& Chong, C. W. (2010). Parametric study of the swimming performance of a fish robot propelled by a flexible caudal fin. Bioinspiration and Biomimetics, 5, 46002.

9. Du, R., Li, Z., Youcef-Toumi, K., Valdivia, Y., \& Alvarado, P. (2015). Robot fish: Bio-inspired fishlike underwater robots. Springer-Verlag.
10. Ebel, G. (2013). Fischschutz und fischabstieg an wasserkraftanlagen: handbuch rechen-und bypasssysteme, gewasserokologie und fischereibiologie Dr. Ebel, $3^{\text {rd }}$ ed, Halle (Saale), Germany. (in German)

11. Cen, L., \& Erturk, A. (2013). Bio-inspired aquatic robotics by untethered piezohydroelastic actuation. Bioinspiration and Biomimetics, 8, 16006.

12. Behbahani, S. B., \& Tan, X. (2016). Design and modeling of flexible passive rowing joint for robotic fish pectoral fins. IEEE Transaction on Robotics, 32, 1119-1132.

13. Wu, Z. X., Yu, J., Tan, M., \& Zhang, J. (2014). Kinematic comparison of forward and backward swimming and maneuvering in a self-propelled sub-carangiform robotic fish. Journal of Bionic Engineering, 11, 199-212.

14. Ay, M., Korkmaz, D., Koca, O. G., Bal, K., Akpolat, Z. H., \& Bingol, M. C. (2018). Mechatronic design and manufacturing of the intelligent robotic fish for bio-inspired swimming modes. Electronics, 7, 118

15. Wang, W., Gu, D., \& Xie, G. (2017). Autonomous optimization of swimming gait in a fish robot with multiple onboard sensors. IEEE Transactions on Systems, Man, and Cybernetics, 49, 891-903.

16. Zhang, S., Qian, Y., Liao, P., Qin, F., \& Yang, J. (2016). Design and control of an agile robotic fish with integrative biomimetic mechanisms. IEEE/ASME Transactions on Mechatronics, 21, $1846-1857$.

17. Liu, B., \& Hammond, F. L. (2020) Modular platform for the exploration of form-function relationships in soft swimming roots. 3rd IEEE International Conference on Soft Robotics (RoboSoft), New Haven, USA, 772-778.

18. Kodati, P., Hinkle, J., Winn, A., \& Deng, X. (2008). Microautonomous robotic ostraciiform (MARCO): Hydrodynamics, design, and fabrication. IEEE Transactions on Robotics, 24, 105-117.

19. Wu, Z., Yu, J., Yuan, J., \& Tan, M. (2019). Towards a gliding robotic dolphin: Design, modeling, and experiments. IEEE/ASME Transactions on Mechatronics, 24, 260-270.

20. Zheng, X., Wang, W., Xiong, M., \& Xie, G. (2020). Online state estimation of a fin-actuated underwater robot using artificial lateral line system. IEEE Transactions on Robotics, 36, 472-487.

21. Hu, Y., Liang, J., \& Wang, T. (2015). Mechatronic design and locomotion control of a robotic thunniform swimmer for fast cruising. Bioinspiration and Biomimetics, 10, 26006.

22. Zuo, W., Keow, A., Chen, Z. (2019). Three-dimensionally maneuverable robotic fish enabled by servo motor and water electrolyser. International Conference on Robotics and Automation (ICRA), Montreal, Canada, 4667-4673.

23. Du, S., Wu, Z., \& Yu, J. (2019). Design and yaw control of a two-motor-actuated biomimetic robotic fish. IEEE International Conference on Robotics and Biomimetics (ROBIO), Sanya, China, 126-131.

24. Marchese, A. D., Onal, C. D., \& Rus, D. (2014). Autonomous soft robotic fish capable of escape maneuvers using fluidic elastomer actuators. Soft Robotics, 1, 75-87.

25. Shaw, H., \& Thakur, A. (2019). Shape memory alloy based caudal fin for a robotic fish: Design, fabrication, control and characterization. Proceedings of the Advances in Robotics, Chennai, India, $1-6$.

26. Gupta, U., Qin, L., Wang, Y., Godaba, H., \& Zhu, J. (2019). Soft robots based on dielectric elastomer actuators: A review. Smart Material StructurE, 28, 103002.

27. Hubbard, J. J., Fleming, M., Palmre, V., Pugal, D., Kim, K. J., \& Leang, K. K. (2013). Monolithic IPMC fins for propulsion and maneuvering in bioinspired underwater robotics. IEEE Journal of Oceanic Engineering, 39, 540-551. 
28. Shintake, J., Cacucciolo, V., Shea, H., \& Floreano, D. (2018). Soft biomimetic fish robot made of dielectric elastomer actuators. Soft Robotics, 5, 466-474.

29. Berlinger, F., Duduta, M., Gloria, H., Clarke, D., Nagpal, R., \& Wood, R. (2018) A modular dielectric elastomer actuator to drive miniature autonomous underwater vehicles. IEEE International Conference on Robotics and Automation (ICRA), Xi' an, China, 3429-3435.

30. Macro fiber composite (mfc) brochure, [2021-09-17], https:// www.smart-material.com/media/Datasheets/MFC_V2.4-datas heet-web.pdf

31. Chen, D., Shao, W., \& Xu, C. (2018). Development of a soft robotic fish with BCF propulsion using MFC smart materials. 37th Chinese Control Conference (CCC), Wuhan, China, 5358-5363.
32. Lou, B., Ni, Y., Mao, M., Wang, P., \& Cong, Y. (2017). Optimization of the kinematic model for biomimetic robotic fish with rigid headshaking mitigation. Robotics, 6, 30 .

33. Lighthill, M. J. (1971). Large-amplitude elongated-body theory of fish locomotion. Proceedings of the Royal Society of London. Series B, Biological Sciences, 179, 125-138.

34. Lagarias, J. C., Reeds, J. A., Wright, M. H., \& Wright, P. E. (1998). Convergence properties of the Nelder-Mead simplex method in low dimensions. Society for Industrial and Applied Mathematics Journal on Optimization, 9, 112-147.

Publisher's Note Springer Nature remains neutral with regard to jurisdictional claims in published maps and institutional affiliations. 